\title{
Bone marrow derived mesenchymal stem cells pretreated with erythropoietin accelerate the repair of acute kidney injury
}

Song Zhou ${ }^{\dagger}$, Yu-ming Qiao ${ }^{\dagger}$, Yong-guang Liu ${ }^{\dagger}$, Ding Liu, Jian-min Hu, Jun Liao, Min Li, Ying Guo, Li-pei Fan, Liu-Yang Li and Ming Zhao*

\begin{abstract}
Background: Mesenchymal stem cells (MSCs) represent a promising treatment option for acute kidney injury (AKI). The main drawbacks of MSCs therapy, including the lack of specific homing after systemic infusion and early cell death in the inflammatory microenvironment, directly affect the therapeutic efficacy of MSCs. Erythropoietin (EPO)preconditioning of MSCs promotes their therapeutic effect, however, the underlying mechanism remains unknown. In this study, we sought to investigate the efficacy and mechanism of EPO in bone marrow derived mesenchymal stem cells (BMSCs) for AKI treatment.

Results: We found that incubation of BMSCs with ischemia/reperfusion(I/R)-induced AKI kidney homogenate supernatant (KHS) caused apoptosis in BMSCs, which was decreased by EPO pretreatment, indicating that EPO protected the cells from apoptosis. Further, we showed that EPO up-regulated silent information regulator 1 (SIRT1) and BCl-2 expression and down-regulated p53 expression. This effect was partially reversed by SIRT1 siRNA intervention. The anti-apoptotic effect of EPO in pretreated BMSCs may be mediated through the SIRT1 pathway. In a rat AKI model, $24 \mathrm{~h}$ after intravenous infusion, GFP-BMSCs were predominantly located in the lungs. However, EPO pretreatment reduced the lung entrapment of BMSCs and increased their distribution in the target organs. AKI rats infused with EPO-BMSCs had significantly lower levels of serum IL-1 $\beta$ and TNF- $a$, and a significantly higher level of IL-10 as compared to rats infused with untreated BMSCs. The administration of EPO-BMSCs after reperfusion reduced serum creatinine, blood urea nitrogen, and pathological scores in I/R-AKI rats more effectively than BMSCs treatment did.
\end{abstract}

Conclusions: Our data suggest that EPO pretreatment enhances the efficacy of BMSCs to improve the renal function and pathological presentation of I/R-AKI rats.

Keywords: Acute kidney injury, Bone marrow derived mesenchymal stem cells, Erythropoietin, Lung entrapment, SIRT1

${ }^{*}$ Correspondence: zhaoming02@hotmail.com

†Song Zhou, Yu-ming Qiao and Yong-guang Liu contributed equally to this work

Department of Organ Transplantation, Zhujiang Hospital, Southern Medical University, No. 253, Industrial Avenue, Haizhu District,

Guangzhou 510280, Guangdong Province, China

\begin{abstract}
Background
Acute kidney injury (AKI) is one of the most clinically impactful diseases with high morbidity and mortality $[1$, 2]. Multiple injuries such as those resulting from sepsis, ischemia/reperfusion ( $/ / R$ ), and drug administration may induce AKI. I/R injury is a major cause of human AKI, which is associated with tubular necrosis, cast formation, tubular dilation, loss of brush border, and inflammation [3]. AKI remains a worldwide public health concern
\end{abstract}

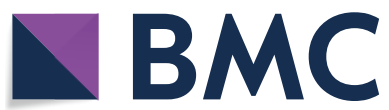

(c) The Author(s) 2020. This article is licensed under a Creative Commons Attribution 4.0 International License, which permits use, sharing, adaptation, distribution and reproduction in any medium or format, as long as you give appropriate credit to the original author(s) and the source, provide a link to the Creative Commons licence, and indicate if changes were made. The images or other third party material in this article are included in the article's Creative Commons licence, unless indicated otherwise in a credit line to the material. If material is not included in the article's Creative Commons licence and your intended use is not permitted by statutory regulation or exceeds the permitted use, you will need to obtain permission directly from the copyright holder. To view a copy of this licence, visit http://creativecommons.org/licenses/by/4.0/. The Creative Commons Public Domain Dedication waiver (http://creativecommons.org/publicdomain/zero/1.0/) applies to the data made available in this article, unless otherwise stated in a credit line to the data. 
due to the increased risk of subsequent development of chronic kidney disease (CKD) [4]. The annual medical expenses associated with AKI treatment places a heavy burden on the public health care system, and yet AKI lacks an established treatment strategy. Therefore, there is an urgent need to find innovative and effective therapeutic strategies for treating AKI. The application of mesenchymal stem cells (MSCs) has been suggested as a promising treatment strategy for AKI $[5,6]$.

In recent years, MSCs have become an area of intense research in the field of stem cell therapy. MSCs are adherent, fibroblast-like cells derived from different tissues and organs, including bone marrow, umbilical cord blood, adipose tissue, and solid organs that have the potential for multidirectional differentiation and self-renewal. The tissue reparative/regenerative and immunoregulatory properties of MSCs render them as promising candidates for cell therapy and tissue regeneration. A large number of basic science studies and clinical trials have demonstrated the safety, feasibility, and effectiveness of MSCs in the treatment of myocardial infarction, spinal cord injury, diabetes, and kidney disease [7-9]. MSCs exhibit renoprotective effects in both acute and chronic kidney injury. These effects seem to be associated with immunomodulation, anti-apoptotic effects, and reduction of disease-related inflammation $[10,11]$.

The main drawbacks of MSCs therapy are early pulmonary entrapment and the lack of specific homing to target tissues following systemic infusion [12, 13]. Even when the MSCs reach the target organ, many of the cells undergo apoptosis mainly due to the inhospitable local microenvironmental conditions, such as hypoxia, oxidative stress, and inflammation, which directly affect the therapeutic efficacy of MSCs $[14,15]$. Application of pretreated MSCs is a novel strategy to enhance the capacity of MSCs to migrate and promote tissue repair in AKI [16, 17].

EPO is a glycoprotein hormone that promotes the proliferation and differentiation of bone marrow hematopoietic stem cells (HSCs) and the hematopoietic function of bone marrow through EPO receptor (EpoR) signaling. BMSCs and bone marrow HSCs are homologous and express EpoR. Studies have shown that EPO is suitable for the treatment of a variety of diseases, including cerebral ischemia, myocardial infarction, chronic congestive heart failure, and renal injury $[18,19]$. In our previous study, we demonstrated that pretreatment with $500 \mathrm{IU} /$ $\mathrm{ml}$ erythropoietin (EPO) for $48 \mathrm{~h}$ prior to infusion markedly increased the homing and healing abilities of bone marrow-derived mesenchymal stem cells (BMSCs). These BMSCs significantly inhibited the apoptosis induced by cyclosporine A (CsA) toxicity in HK2 cells. Moreover, the single infusion treatment with EPO-BMSCs significantly improved the renal function in CsA-induced chronic toxic renal injury, and promoted the repair of renal fibrosis in rats [20].

In this study, we sought to investigate the efficacy and mechanism of EPO pretreatment on BMSCs for the treatment of AKI. We demonstrate that EPO promotes the survival of transplanted BMSCs in an inflammatory microenvironment by stimulating the SIRT1-dependent pathway.

\section{Results}

The characterization of EPO-treated BMSCs

The passage 4 (P4) BMSCs and EPO-treated BMSCs were observed under the phase contrast microscope (Fig. 1a). Both untreated and EPO-treated BMSCs were capable of osteogenic and adipogenic differentiation when cultured in the appropriate inducing media. After 3 weeks of incubation, BMSCs and EPO-BMSCs differentiated into osteoblasts and adipocytes (Fig. 1b). Flow cytometric analysis confirmed that both groups of cells were CD45-negative but positive for the phenotypic markers CD90 and CD44 (Fig. 1c). Both groups of cells maintained their stem cell characteristics.

\section{EPO-pretreated inhibited AKI-KHS-induced apoptosis in BMSCs}

To study the effect of EPO on BMSCs, we established AKI-KHS-induced in vitro injury model. After co-culture with AKI-KHS for $24 \mathrm{~h}$, the EPO-pretreated attenuated the apoptosis rate of BMSCs significantly. BMSCs, EPOBMSCs were incubated with AKI-KHS (or N-KHS), after culture for $24 \mathrm{~h}$, flow cytometric analysis was performed to assess apoptosis in each group (Fig. 2a). The apoptotic rates were significantly higher in the BMSCs + AKIKHS and EPO-BMSCs + AKI-KHS groups when compared with that of the control group $(\mathrm{p}<0.05)$. AKI-KHS induced apoptosis in BMSCs, but EPO pretreatment protected the cells from this apoptotic effect $(\mathrm{p}<0.05)$ (Fig. 2b).

Protein was extracted from cultured cells and examined using western blot analyses (Fig. 2c). SIRT1 and Bcl-2 protein expression in both BMSCs + AKI-KHS and EPOBMSCs + AKI-KHS groups were significantly increased compared to that in the control group $(\mathrm{p}<0.05)$, especially in the EPO-BMSCs + AKI-KHS group $(\mathrm{p}<0.05)$. The expression of $\mathrm{p} 53$ protein was significantly higher in the BMSCs + AKI-KHS group compared to the other two groups $(\mathrm{p}<0.05)$.

\section{EPO exerted protective effect on BMSCs subjected AKI-KHS via activating SIRT1-p53 signaling}

Results of the flow cytometric analysis demonstrated that AKI-KHS-treated had an adverse effect 


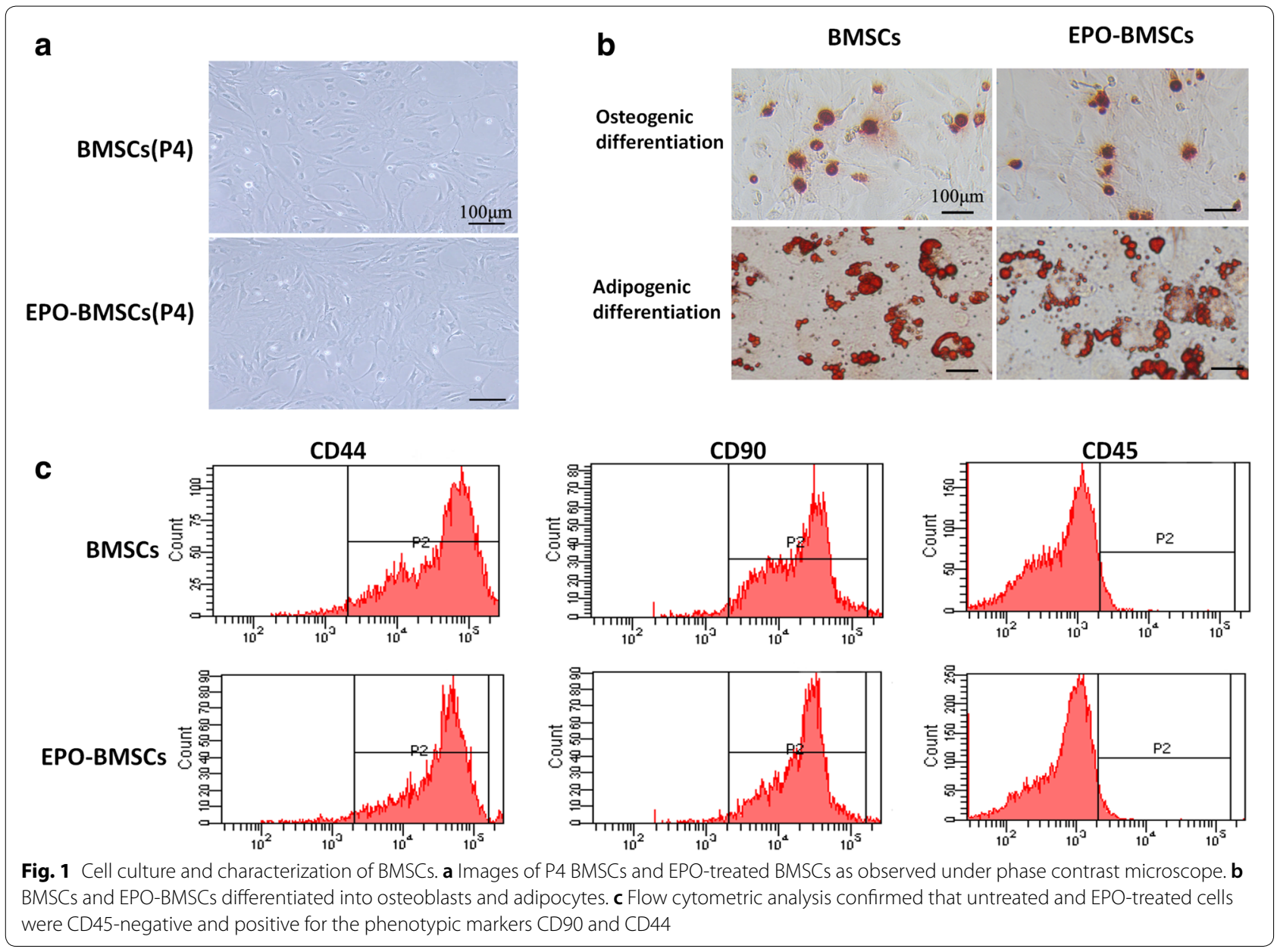

on the survival of BMSCs while the EPO pretreatment improved BMSCs survival in the AKI microenvironment. To further investigate the anti-apoptotic mechanism of EPO pretreatment of the BMSCs, we analyzed the expression of the apoptotic factor p53, and SIRT1. The expression of SIRT1 in the EPOBMSCs + AKI-KHS group was significantly increased compared with that in the BMSCs + AKI-KHS group $(\mathrm{p}<0.05)$, and the expression of p53 was significantly higher in the BMSCs + AKI-KHS group than in the EPO-BMSCs + AKI-KHS group $(p<0.05)$. The effect of EPO on the expression of SIRT1 and p53 was partially reversed by SIRT1 siRNA intervention (Fig. 3a-c).

To prove the anti-apoptotic effect of EPO on BMSCs though SIRT1, we established AKI-KHS-induced in vitro injury model again. BMSCs, EPO-BMSCs, EPOBMSCs + SIRT1 siRNA, and EPO-BMSCs + Con siRNA were incubated with AKI-KHS, and BMSCs incubated with N-KHS was used as control. After culture for $24 \mathrm{~h}$, flow cytometric analysis was performed to assess apoptosis in each group (Fig. 3d). The anti-apoptotic effect of
EPO was partially reversed by SIRT1 siRNA intervention (Fig. 3e).

\section{Pretreatment with EPO markedly increased the homing abilities of BMSCs}

In our previous study, we demonstrated that pretreatment with $500 \mathrm{IU} / \mathrm{ml}$ EPO for $48 \mathrm{~h}$ prior to infusion markedly increased the homing abilities of BMSCs. To verify the in vivo distribution of BMSCs after intravenous infusion, we prepared fast-frozen sections from the kidney, lung, spleen, and liver $24 \mathrm{~h}$ after GFP-BMSCs and GFP-EPO-BMSCs infusion. Our data showed that $24 \mathrm{~h}$ after intravenous infusion, a majority of the GFP-BMSCs were trapped in the lungs, with minimal fluorescence detected in the spleen, liver, and kidneys. In the GFPEPO-BMSCs group, the fluorescence intensity was significantly lower in the lungs but higher in other organs, especially the kidneys, compared with the corresponding fluorescence intensities in the GFP-BMSCs group. GFP-BMSCs and GFP-EPO-BMSCs were predominantly detected in the lungs, but EPO preconditioning reduced 

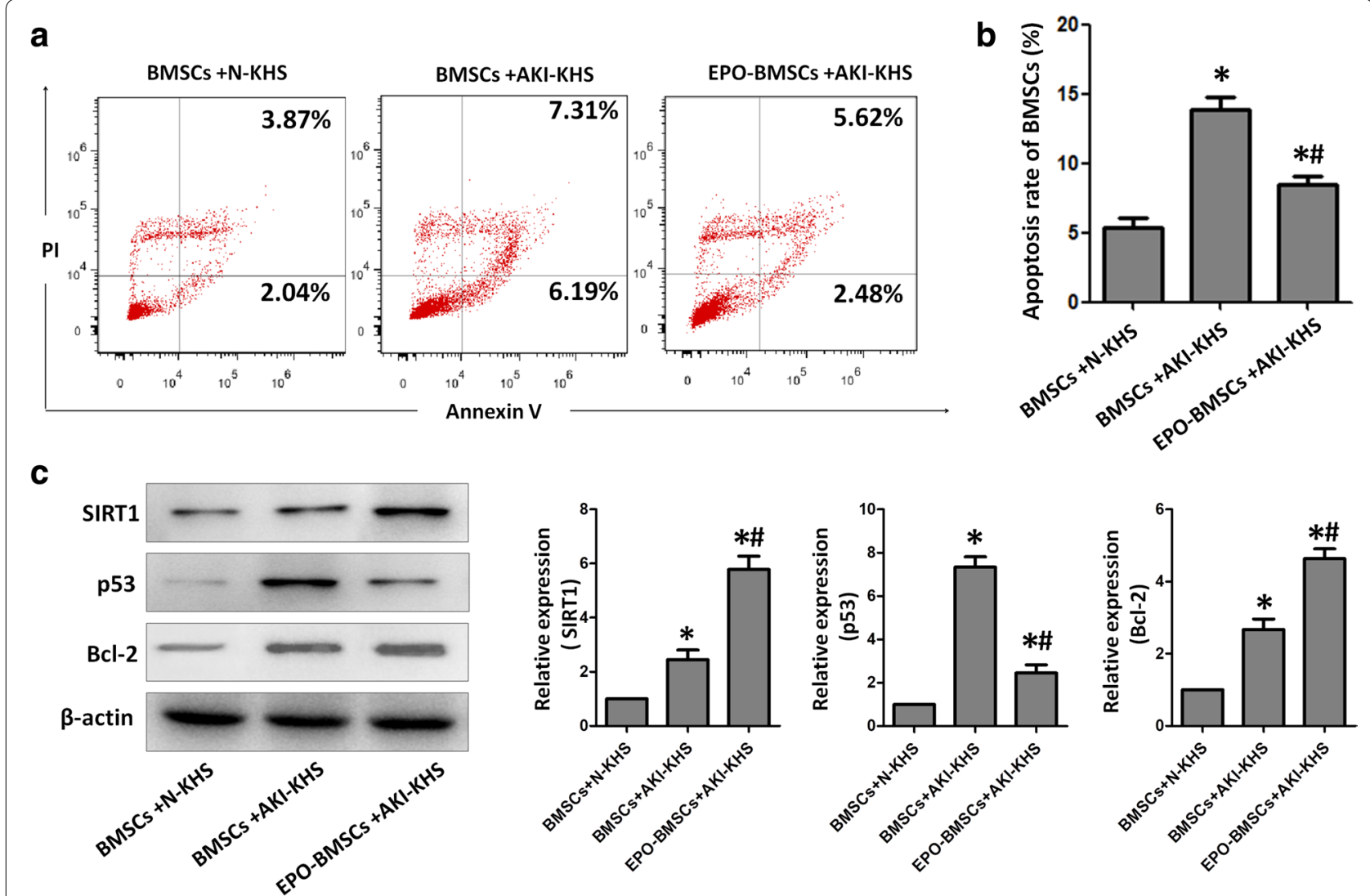

Fig. 2 AKI-KHS induced apoptosis in BMSCs. a Flow cytometric analysis was performed $24 \mathrm{~h}$ after culturing to assess apoptosis in each group. b Apoptotic rates in each group were analyzed. c Anti-apoptotic factor Bcl-2, apoptotic factor p53, and SIRT1 were examined using western blot analyses. ${ }^{*} p<0.05$ versus BMSCs $+\mathrm{N}-\mathrm{KHS}, \# \mathrm{p}<0.05$ versus BMSCs$+\mathrm{AKI}-\mathrm{KHS}$

the lung entrapment of BMSCs and increased their distribution in the target organs (Fig. 4).

\section{EPO-BMSCs improved renal function after I/R injury}

To compare the effects of BMSCs and EPO-BMSCs on AKI rats, we measured the levels of blood urea nitrogen (BUN) and serum creatinine (SCr) on day 1 and 5 after treatment. Treatment of AKI rats with EPO, BMSCs, and EPO-BMSCs showed varying therapeutic effects. All three treatment groups reduced the level of BUN and $\mathrm{SCr}$ on day 1 and 5 compared with the model group. However, only BMSCs and EPO-BMSCs treatment showed a significant effect $(\mathrm{p}<0.05)$. Rats in the EPO-BMSCs group had significantly lower BUN and $\mathrm{SCr}$ levels than those in the BMSCs group on day 5 ( $p<0.05$, Fig. $5 a, b)$.

\section{EPO-BMSCs suppressed inflammatory response after I/R injury}

Twenty-four hours after the treatment of AKI rats, serum concentrations of the inflammatory factors were measured by ELISA. There was a significant increase in the level of IL- $1 \beta$, TNF- $\alpha$, and IL-10 1 day after the induction of AKI. AKI rats that underwent EPO-BMSCs infusion had significantly lower serum IL- $1 \beta$ and TNF- $\alpha$ levels and a significantly higher IL-10 serum level than those in AKI rats that received BMSCs infusion. The serum levels of the cytokines in the EPO group did not significantly differ from those in the model group (Fig. $5 \mathrm{c}-\mathrm{e}$ ).

\section{EPO-BMSCs enhanced the effect to ameliorate pathological injuries}

Hematoxylin and eosin (HE) staining of kidney sections revealed tubular necrosis, cast formation, tubular dilation, and loss of brush border in the AKI model group (Fig. 6a). These pathological injuries were assessed using the pathological scores obtained after treatment with EPO, BMSCs, and EPO-BMSCs on day 1 and 5 . The pathological scores in the BMSCs and EPO-BMSCs treatment groups were significantly lower than those in the model group 1 and 5 days after treatment $(\mathrm{p}<0.05)$. Both BMSCs and EPO-BMSCs infusion reduced tubular injury; however, EPO-BMSCs administration improved tubular injury significantly more on day 5 as compared to infusion with BMSCs (Fig. 6b). 


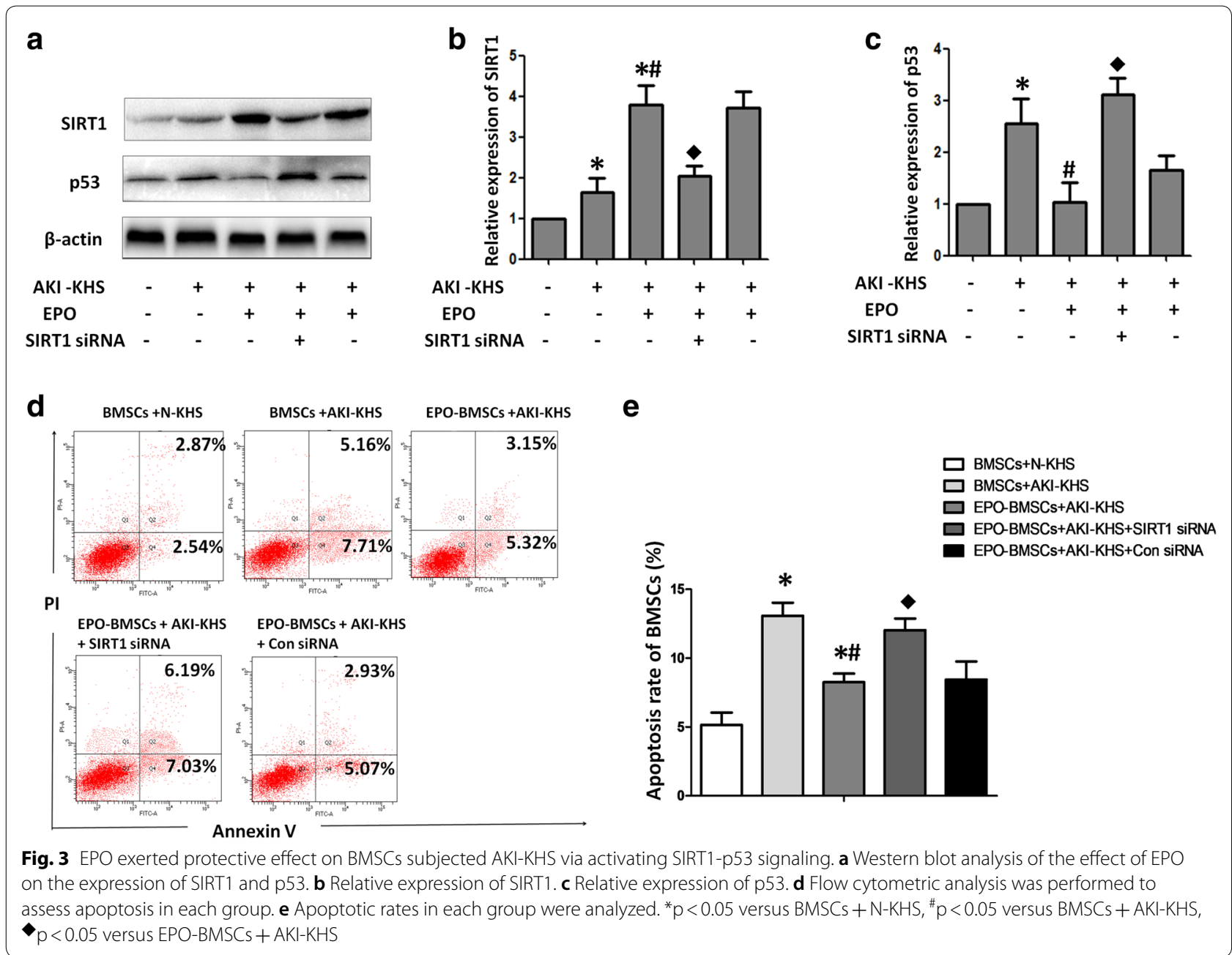

\section{Discussion}

In the present study, we reveal novel insights regarding the efficacy and mechanism of EPO-BMSCs in I/R-AKI. Our findings showed that I/R-AKI microenvironment caused apoptosis in BMSCs, but EPO pretreatment protected the cells from this apoptotic effect. We found that EPO up-regulated SIRT1 and Bcl-2 expression and down-regulated p53 expression in the AKI model. The anti-apoptotic effect of EPO may be mediated through the SIRT1 pathway. EPO pretreatment reduced pulmonary entrapment of BMSCs and increased the number of cells reaching the target organs. Furthermore, EPO pretreatment reduced the expression of disease-related inflammatory cytokines. AKI rats that underwent EPOBMSCs infusion had significantly lower serum IL-1 $\beta$ and TNF- $\alpha$ levels, and a significantly higher IL-10 level than those in rats treated with BMSCs. Our data suggest that EPO pretreatment enhances the efficacy of BMSCs to improve renal function and pathological presentation of I/R-AKI rats.
The protective effect of MSCs in acute and chronic renal injury may be through a paracrine/autocrine mechanism, which is related to immune regulation, antiapoptosis, and reduction of disease-related inflammation. Several studies have shown that the survival and retention of MSCs in target organs or tissues is closely related to the therapeutic effect mediated by MSCs. The main drawbacks of MSCs therapy are the early pulmonary entrapment and the lack of specific homing after systemic infusion. Most of the MSCs undergo cell death after transplantation, mainly due to an adverse microenvironment, directly affecting the therapeutic efficacy of MSCs $[14,15]$.

Increasing the dose of MSCs infused represents a viable option to achieve better therapeutic effects; however, this is accompanied by some side effects, such as microvascular embolization and the potential risk of long-term tumor growth. Therefore, current studies on MSCsrelated therapies have focused on lower infusion doses to achieve the greatest therapeutic effect possible. Cell-free 


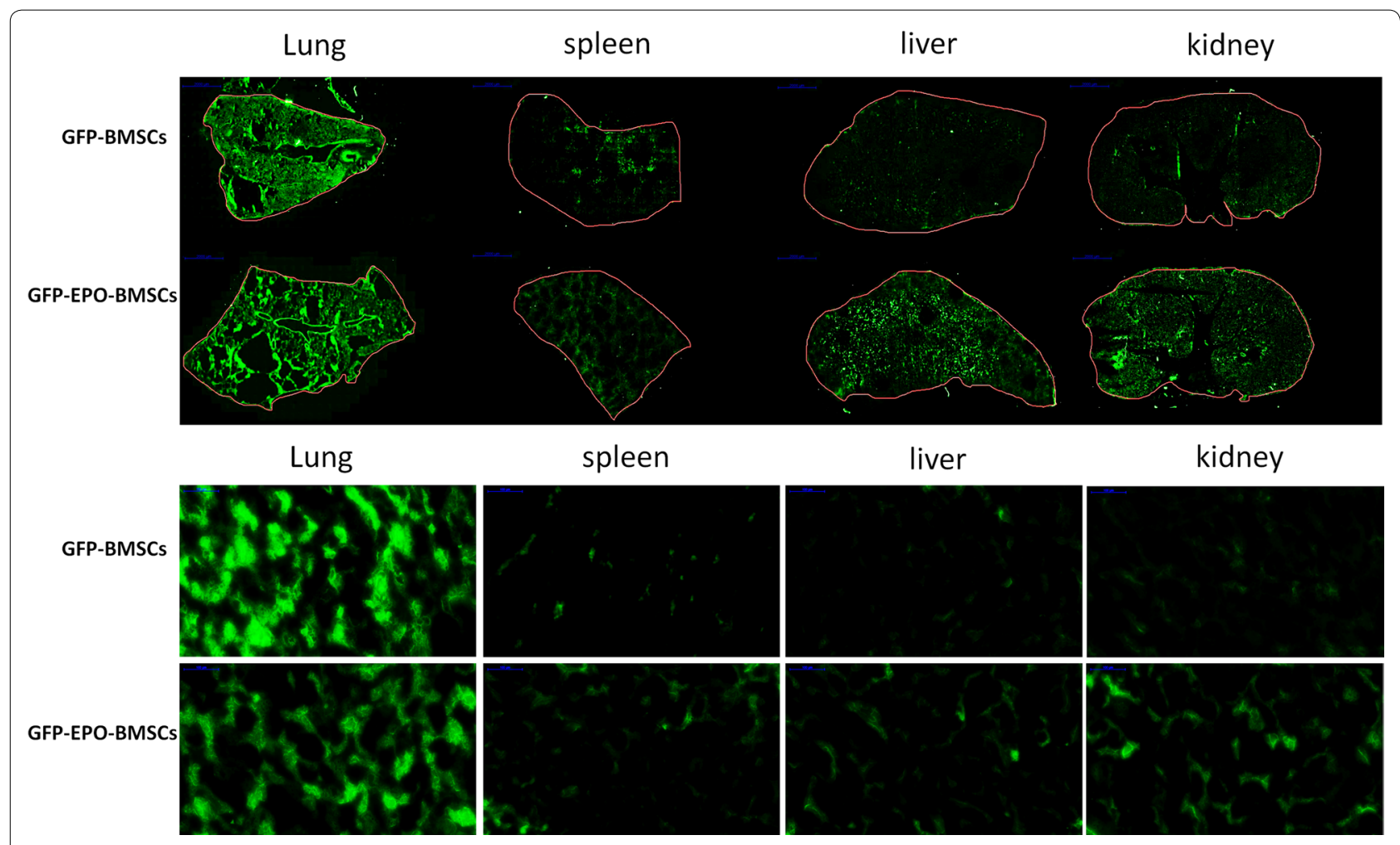

Fig. 4 GFP fluorescence images of frozen sections from the lung, spleen, liver and kidney of rats. Sections were prepared $24 \mathrm{~h}$ after intravenous infusion of BMSCs and EPO-BMSCs

treatments, including microvesicles, exosomes, specific cytokines, miRNA, and the pretreatment of MSCs, represent viable alternatives to address the issue of long-term negative effects [21-23]. Pretreating MSCs is a novel strategy to enhance the capacity of MSCs to migrate and promote tissue repair in injured kidneys [24]. Pretreatment, before infusion, with cytokines such as transforming growth factor $\beta 1$ (TGF- $\beta 1$ ), interleukin-17A (IL-17A), and melatonin can increase the number of MSCs homing to the injured kidney, promote the recovery of renal function, and ameliorate renal impairments [3, 17, 25]. Similar results were obtained upon pretreating MSCs with EPO.

EPO is a glycoprotein hormone that promotes the proliferation and differentiation of bone marrow HSCs and the hematopoietic function of bone marrow through EpoR signaling. BMSCs and bone marrow HSCs are homologous and express EpoR. However, many other cell types, including neurons, endothelial cells, cardiomyocytes, and renal tubular cells also express the EpoR and respond to EPO treatment [26]. Studies have shown that EPO is suitable for the treatment of a variety of diseases, including cerebral ischemia, myocardial infarction, chronic congestive heart failure, and renal injury $[18,19]$. Moreover, some studies suggest that overexpression of EPO in MSCs by gene-transfection could further enhance the therapy effect of MSCs [27, 28]. Compared with other pretreatments or strategies that improve the therapeutic effect of MSCs, the in vitro pretreatment with EPO has significant advantages in terms of clinical feasibility. First, EPO is a commonly used therapeutic drug with few side effects and is widely used in the clinical treatment of anemia, especially in patients with CKD. Second, it exhibits anti-oxidative and anti-inflammatory effects.

Our data showed that $24 \mathrm{~h}$ following intravenous infusion, GFP-BMSCs were predominantly located in the lungs. EPO preconditioning reduced the entrapment of BMSCs in the lungs and increased the distribution of GFP-BMSCs in the target organs. In our previous study, we showed that the pretreatment of BMSCs with an optimal concentration of EPO for an appropriate time induced a marked change in the proliferation rate and cytoskeletal rearrangement of BMSCs. After incubation with EPO, most of the cells exhibited parallelly-oriented filaments organized along the cell axis. We observed that C-X-C chemokine receptor type 4 (CXCR4), a pivotal mediator of migration and engraftment in MSCs, was up-regulated following EPO treatment, enhancing the migration ability of BMSCs [20]. 


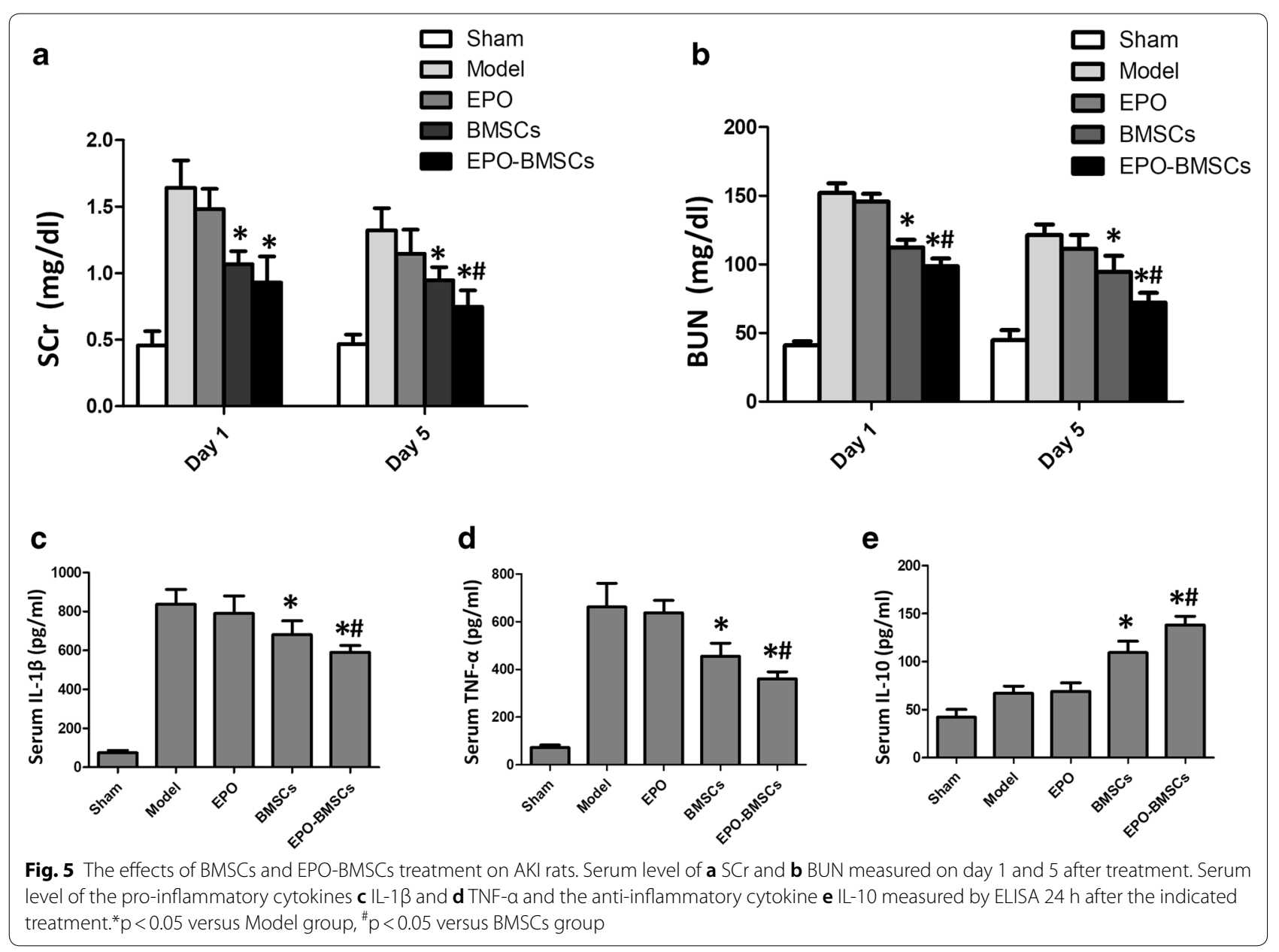

Homing of MSCs to injured tissues is very critical in cell therapy. Various methods to apply MSCs exist, such as peripheral intravenous infusion, arterial infusion to the target organ, and local injection. Local injection increases the risk of bleeding and tissue injury, while direct arterial administration can result in occlusion and embolization of the target organs. Therefore, MSCs are mostly administered through a standard intravenous route. Pulmonary entrapment is a major problem after intravenous infusion of MSCs. Harting et al. [29] demonstrated that less than $4 \%$ of the infused cells were likely to traverse the pulmonary microvasculature and reach the arterial circulation, a phenomenon termed "pulmonary first-pass effect", which limits the efficacy of this therapeutic approach. Some studies show that small microspheres $(4-5 \mu \mathrm{m})$ could pass through the pulmonary system, whereas the majority of the large microspheres $(20-\mu \mathrm{m})$ and MSCs $(15-19 \mu \mathrm{m})$ are trapped within the lungs. Lung entrapment may be due to the small capillary size, large capillary network of the lungs, and strong adhesion properties of MSCs [30-32]. A variety of molecules may be involved in lung entrapment of systemically infused cells, and the composition of cell surface molecules, such as $\alpha 4, \alpha 5$, and $\alpha 6$ integrins, may affect the migratory behavior of the therapeutic cells $[13,32]$. We found that after intravenous infusion, most of the stem cells were trapped within the lungs, but EPO pretreatment reduced pulmonary entrapment and increased the number of cells reaching the target organ.

We also found that EPO up-regulated SIRT1 and Bcl-2 expression and down-regulated p53 expression. SIRT1 is an $\mathrm{NAD}^{+}$-dependent deacetylase belonging to class III histone deacetylases and is known as the longevity protein in mammals. SIRT1 regulates a variety of cellular signaling pathways by modifying the acetylation status of target proteins including $\mathrm{p} 53$, members of the forkhead family of transcription factors (FOXO), and nuclear factor NF- $\mathrm{kB}$. Following activation, SIRT1 participates in a plethora of cellular processes such as cell senescence, apoptosis, DNA damage repair, cell cycle, oxidative stress response, energy metabolism regulation, tumor generation, and other physiological and pathological processes 


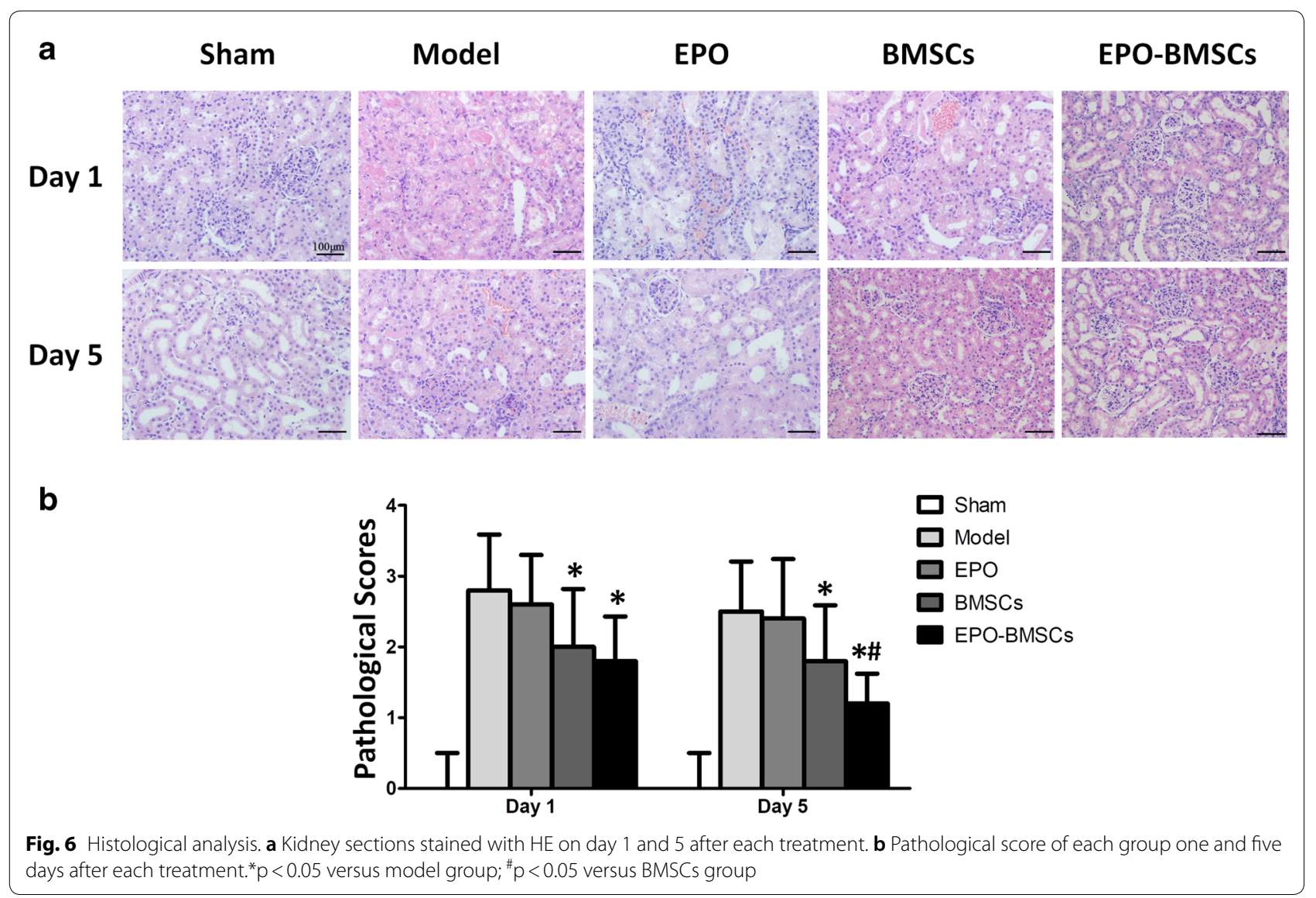

[33-35]. Tumor suppressor protein, p53, plays a vital role in apoptotic signaling pathways, including membrane and mitochondrial apoptotic pathways, and affects the transcription and expression of many apoptosis-related cytokines in the nucleus [36]. SIRT1 reduces the transcriptional activity of p53 and inhibits p53-dependent cell apoptosis, caused by DNA damage [37]. In tumor studies, SIRT1 inhibited apoptosis in tumor cells by regulating p53 and Bcl-2 [38]. EPO has been demonstrated to impede DOX-induced cardiotoxicity by activating SIRT1, leading to enhancement of mitochondrial function [18]. Hong et al. [33] revealed that EPO alleviates hepatic steatosis by activating autophagy through SIRT1-dependent de-acetylation of LC3. SIRT1 plays an important role in maintaining the self-renewal and differentiation of MSCs, especially under stress conditions [39, 40], and also exhibits positive effects on senescence and apoptosis of MSCs [41, 42].

In our study, most of the BMSCs undergo cell death after transplantation, mainly due to the adverse local microenvironment. Therefore, improving cell survival and retention of BMSCs is pertinent to promote their therapeutic efficacy in AKI therapy. The observed beneficial effect of EPO pretreatment was associated with SIRT1 signaling activation, leading to the deacetylation and subsequent inactivation of p53 and upregulation of $\mathrm{Bcl}-2$ expression, which in turn reduces apoptosis in BMSCs. These findings, therefore, demonstrate that the anti-apoptotic effect following EPO pretreatment of BMSCs may be mediated though the SIRT1 pathway.

The mechanism by which EPO-pretreated BMSCs accelerate the repair of AKI includes three facets. First, after incubation with EPO, most of the BMSCs exhibited parallelly oriented filaments organized along the cell axis and showed increased CXCR4 expression. These changes reduced the lung entrapment of BMSCs and increased their homing to target organs. Second, EPO up-regulated SIRT1 and Bcl-2 expression, and down-regulated p53 expression in BMSCs. SIRT1 inhibited apoptosis in BMSCs by regulating p53 and Bcl-2 expression. Third, the direct anti-inflammatory effect of EPO-BMSCs is also likely to be involved in the process. However, the present study has some limitations and did not elucidate the mechanism underlying EPO-mediated activation of SIRT1 signaling in BMSCs, which requires future investigations. 


\section{Conclusion}

In conclusion, EPO-BMSCs were more effective in reducing the levels of $\mathrm{SCr}$ and $\mathrm{BUN}$, and the pathological scores in I/R-AKI rats after reperfusion when compared to untreated BMSCs. These results suggest that EPO pretreatment may potentially be a novel alternative to untreated BMSCs for the management of AKI.

\section{Materials and methods \\ Cell culture}

Sprague-Dawley (SD) bone marrow derived mesenchymal stem cells (BMSCs) (Cyagen Biosciences Inc., Guangzhou, China) were propagated at $37{ }^{\circ} \mathrm{C}$ under $5 \%$ $\mathrm{CO}_{2}$ in Dulbecco's Modified Eagle Medium (DMEM, Gibco, Carlsbad, CA) cultured with $10 \%$ fetal bovine serum (FBS, Gibco) and $1 \%$ antibiotics $\left(50 \mathrm{U} \mathrm{mL}^{-1}\right.$ pen-

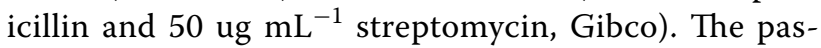
sage $4(\mathrm{P} 4)$ cells were used for the in vitro experiments. SD-derived BMSCs(P3) (Cyagen Biosciences Inc., USA) that stably express GFP were propagated and the P5-GFP-BMSCs were used for in vivo experiments.

\section{Characterization of BMSCs incubated with EPO}

BMSCs were trypsinized after incubation with or without EPO $(500 \mathrm{IU} / \mathrm{ml})$ for $48 \mathrm{~h}$ and washed three times with phosphate-buffered saline (PBS). Cell surface markers were examined by immunostaining with the following antibodies: phycoerythrin (PE)-conjugated anti-CD45 (BD Biosciences, San Jose, CA), fluorescein isothiocyanate (FITC)-conjugated anti-CD44 (BD Biosciences), and FITC-conjugated anti-CD90 (BD Biosciences) antibody. The labeled cells were analyzed using a flow cytometer (BD LSRFortessa ${ }^{\mathrm{TM}}$, Piscataway, $\mathrm{NJ})$. Osteogenic and adipogenic differentiation potential of the cells in the two groups was examined according to the manufacturer's protocol (Cyagen, China).

I/R-AKI kidney homogenate supernatant (KHS) preparation An AKI model was established using SD rats by clamping both renal pedicles for $45 \mathrm{~min}$, followed by clamp-release to allow reperfusion. Both kidneys were harvested $60 \mathrm{~min}$ after reperfusion, cut into small pieces, and homogenized in PBS using a glass homogenizer to obtain a $20 \mathrm{~g} / 1$ homogenate. The homogenate was then centrifuged at $20,000 \mathrm{rpm}$ for $15 \mathrm{~min}$ at $4{ }^{\circ} \mathrm{C}$. The supernatant was filtered through a $30-\mu \mathrm{m}$ meshsized disposable sterile filter to obtain AKI-KHS, which was stored at $-80{ }^{\circ} \mathrm{C}$ until further use. Normal KHS (N-KHS) obtained from healthy SD rats was used as a control.
AKI-KHS-induced apoptosis in BMSCs

To study the anti-apoptotic effect of BMSCs pretreated with EPO, we established AKI-KHS-induced in vitro injury model: BMSCs or EPO-BMSCs were incubated with AKI-KHS (or N-KHS). Cells were seeded at $4 \times 10^{5}$ cells/well in six-well plates in DMEM/F12 with 2\% FBS. Transwell chambers with $0.4 \mu \mathrm{m}$ pore size polycarbonate filter (Corning Incorporated, NY, USA) were introduced in the wells for the interventions. Three groups were set up: the control group (BMSCs + N-KHS group, BMSCs were plated in the 6-well plates and $1.5 \mathrm{ml} \mathrm{N}$-KHS was added to the upper chamber); the BMSCs + AKI-KHS group (BMSCs were plated in the 6-well plates and $1.5 \mathrm{ml}$ AKI-KHS was added to the upper chamber); and the EPO-BMSCs + AKI-KHS group (EPO-BMSCs were plated in the 6-well plates and $1.5 \mathrm{ml}$ AKI-KHS was added to the upper chamber). All groups were incubated at $37{ }^{\circ} \mathrm{C}$ for $24 \mathrm{~h}$ in a humidified atmosphere with $5 \% \mathrm{CO}_{2}$. Then, apoptosis of the BMSCs and EPO-BMSCs was analyzed by flow cytometry, the Annexin V-FITC apoptosis detection kit (Invitrogen, Frederick, MD, USA) was used to detect apoptotic cells according to the manufacturer's protocol. Then cells were harvested subsequently for western blot analysis.

\section{Western blotting}

After each treatment, the cells were washed twice with PBS, harvested, and the proteins were extracted. The following antibodies were used to analyze protein expression: anti-BCL-2 (1:1000, Santa Cruz, CA), p53 (1:1000, Santa Cruz), SIRT1 (1:1000, Santa Cruz), and anti- $\beta$ actin (1:2000, Bioworld, Shanghai, China). Protein bands were quantified by densitometry using an Alpha Innotech imaging system. Protein levels were normalized to $\beta$-actin expression using Image J analysis software.

\section{SiRNA transfection}

Cells were transiently transfected with SIRT1 siRNA or control siRNA using Lipofectamine RNAiMAX reagent (Life Technologies, Carlsbad, CA) according to the manufacturer's protocol. Cells were analyzed $36 \mathrm{~h}$ after siRNA transfection. The SIRT1 siRNA and control siRNA were synthesized by RiboBio technologies (RiboBio, Guangzhou, China). The sequence of SIRT1 siRNA was as follows: 5'-CACCUGAGUUGGAUGAUAUTT-3' (sense) and $5^{\prime}$-AUAUCAUCCAACUAGGUGTT-3' (antisense).

\section{Anti-apoptotic effect of BMSCs pretreated with EPO}

To study the anti-apoptotic effect of BMSCs pretreated with EPO, we incubated BMSCs and EPO-BMSCs with AKI-KHS (or N-KHS). Five groups were set up: the 
BMSCs $+\mathrm{N}-\mathrm{KHS}$ group was used as a control(BMSCs were plated in 6-well plates and $1.5 \mathrm{ml} \mathrm{N}$-KHS was added to the upper chamber); the BMSCs + AKI-KHS group (BMSCs were plated in 6-well plates and $1.5 \mathrm{ml}$ AKI-KHS was added to the upper chamber); the EPOBMSCs + AKI-KHS group (EPO-BMSCs were plated in 6-well plates and $1.5 \mathrm{ml}$ AKI-KHS was added to the upper chamber); the EPO-BMSCs + AKI-KHS + SIRT1 siRNA group (EPO-BMSCs transfected with SIRT1 siRNA were plated in 6-well plates and $1.5 \mathrm{ml}$ AKIKHS was added to the upper chamber); and the EPOBMSCs + AKI-KHS + Con siRNA group (EPO-BMSCs transfected with control siRNA were plated in 6-well plates and $1.5 \mathrm{ml}$ AKI-KHS was added to the upper chamber). All groups were incubated at $37{ }^{\circ} \mathrm{C}$ for $24 \mathrm{~h}$ in a humidified atmosphere of $5 \% \mathrm{CO}_{2}$. Then cells were harvested for western blot analysis. After that, we established AKI-KHS-induced in vitro injury model again, the apoptosis of the BMSCs and EPO-BMSCs was analyzed by flow cytometry.

\section{Animals}

Adult female SD rats (200-250 g), purchased from the animal house of the Faculty of Medicine at Southern Medical University (Guangzhou, China), were used for the study. Animals were housed under specific pathogenfree conditions with a $12 \mathrm{~h}$ dark-light cycle and supplied with pelleted food and tap water ad libitum. Animals were allowed to acclimatize to the housing conditions for 1 week. All procedures were performed in strict accordance with the Guidelines for Animal Experimentation of Southern Medical University (Guangzhou, China).

\section{Animal grouping and treatment}

AKI models were established using SD rats. The animals were anesthetized by intraperitoneal injection of $2 \%$ sodium pentobarbital $(50 \mathrm{mg} / \mathrm{kg})$. Abdominal incisions were made, and the two renal pedicles were bluntly separated. A microvascular clamp was used to clamp both renal pedicles for $45 \mathrm{~min}$, followed by clamp-release to allow reperfusion. Then, the abdominal incision was closed. Intervention treatments were administered to rats through tail vein injection after reperfusion.

To determine the effects of BMSCs and EPO-BMSCs treatment, we randomly divided the rats $(n=50)$ into the following five groups (10 rats per group): sham group (kidneys of the SD rats were exposed for $45 \mathrm{~min}$ and $1 \mathrm{ml}$ low-glucose DMEM was injected), model group (both renal pedicles were clamped for $45 \mathrm{~min}$ and $1 \mathrm{ml}$ low-glucose DMEM was injected), EPO group (both renal pedicles were clamped for $45 \mathrm{~min}$ and $1 \mathrm{ml}$ EPO (500 IU/ml) was injected), BMSCs group (both renal pedicles were clamped for $45 \mathrm{~min}$ and $1 \times 10^{6}$ BMSCs were injected), and EPO-BMSCs group (both renal pedicles were clamped for $45 \mathrm{~min}$ and $1 \times 10^{6} \mathrm{EPO}-\mathrm{BMSCs}$ were injected). All rats were housed at a favorable temperature and humidity (a temperature of $21{ }^{\circ} \mathrm{C} \pm 2{ }^{\circ} \mathrm{C}$, and a humidity of $55 \% \pm 5 \%$ ) with an unlimited supply of water and food post-surgery. Rats were sacrificed 1 and 5 days after treatment (five rats at each time point). Blood samples and tissues from the kidneys, lung, spleen, and liver were collected for subsequent experiments. Blood samples were collected through the inferior vena cava, and serum was separated and stored at $-80^{\circ} \mathrm{C}$ until use. Both kidneys of each rat were immediately excised and cut into two coronal sections. The sections were fixed in $4 \%$ paraformaldehyde at room temperature. In the BMSCs and EPO-BMSCs group, the lung, spleen, liver, and the remaining part of the kidney were immediately analyzed.

\section{GFP fluorescence in frozen tissue sections}

In our previous study, we demonstrated that pretreatment with $500 \mathrm{IU} / \mathrm{ml}$ EPO for $48 \mathrm{~h}$ prior to infusion markedly increased the homing abilities of BMSCs. To verify the in vivo distribution of BMSCs after intravenous infusion, we prepared fast-frozen sections from the kidney, lung, spleen, and liver $24 \mathrm{~h}$ after GFP-BMSCs and GFP-EPO-BMSCs infusion. We immediately observed the sections under a fluorescence microscope.

\section{Renal functional analysis}

Renal function was estimated using diagnostic kits. SCr was measured using a colorimetric microplate assay based on the Jaffe reaction (Quantichrom Creatinine Assay; BioAssay Systems). BUN was measured using a colorimetric assay kit according to the manufacturer's instructions (Quantichrom Urea Assay; BioAssay Systems).

\section{ELISA}

Serum concentrations of the inflammatory factors IL-1 $\beta$ and TNF- $\alpha$ and the anti-inflammatory cytokine IL-10 were measured by ELISA (R\&D Systems, Minneapolis, MN, USA). The assay was performed according to the manufacturer's instructions.

\section{Renal histological analysis}

To detect kidney injuries, we fixed the samples in $4 \%$ neutral-buffered paraformaldehyde for histological assessment, embedded them in paraffin, and cut them in 3- $\mu \mathrm{m}$-thick slices. The sections were then stained with HE (Servicebio, China). Histological examinations were performed in a blinded manner for acute tubular necrosis (ATN) scores regarding the grading of tubular necrosis, cast formation, tubular dilation, and loss of 
brush border. Ten non-overlapping fields $(\times 200)$ were randomly selected and scored as follows: 0 , no damage; 1 , patchy isolated necrosis $\leq 10 \%$; 2 , tubular necrosis between 10 and 25\%; 3, tubular necrosis between 25 and $50 \%$; 4 , tubular necrosis $>50 \%[3]$.

\section{Statistical analysis}

Results are expressed as mean \pm standard deviation. Student's $t$ test was performed to analyze the differences between two groups. Multiple-group comparison was performed using one-way analysis of variance (ANOVA) test. SPSS 19.0 statistical software was used for statistical analysis. $\mathrm{P}<0.05$ was considered statistically significant.

\section{Acknowledgements}

Not applicable.

\section{Authors' contributions}

SZ designed the overall study, executed the experiments, analyzed data, and wrote the manuscript; YMQ executed the experiments, prepared the manuscript; YGL designed the overall study, supervised the project and edited the manuscript; $D L, J M H, J L, M L, Y G, L P F$, LYL provided suggestions for the project and edited the manuscript. MZ: designed the overall study, analyzed data and final approval of manuscript. All authors read and approved the final manuscript.

\section{Funding}

This work was supported by the Initiative Research Projects of Southern Medical University in 2018 (No. PY2018N049).

\section{Availability of data and materials}

Not applicable.

\section{Ethics approval and consent to participate}

Not applicable.

\section{Consent for publication}

Not applicable.

\section{Competing interests}

The authors declare that they have no competing interests.

Received: 12 March 2020 Accepted: 30 October 2020

Published online: 16 November 2020

\section{References}

1. Chawla LS, Bellomo R, Bihorac A, Goldstein SL, Siew ED, Bagshaw SM, Bittleman D, et al. Acute kidney disease and renal recovery: consensus report of the Acute Disease Quality Initiative (ADQI) 16 Workgroup. Nat Rev Nephrol. 2017;13:241-57.

2. Xu X, Nie S, Liu Z, Chen C, Xu G, Zha Y, Qian J, et al. Epidemiology and clinical correlates of AKI in Chinese hospitalized adults. Clin J Am Soc Nephrol. 2015;10:1510-8.

3. Bai M, Zhang L, Fu B, Bai J, Zhang Y, Cai G, Bai X, et al. IL-17A improves the efficacy of mesenchymal stem cells in ischemic-reperfusion renal injury by increasing Treg percentages by the COX-2/PGE2 pathway. Kidney Int 2018:93:814-25.

4. Liu J, Kumar S, Dolzhenko E, Alvarado GF, Guo J, Lu C, Chen Y, et al. Molecular characterization of the transition from acute to chronic kidney injury following ischemia/reperfusion. JCl insight. 2017;2:e94716.

5. Xiang J, Jiang T, Zhang W, Xie W, Tang X, Zhang J. Human umbilical cordderived mesenchymal stem cells enhanced HK-2 cell autophagy through MicroRNA-145 by inhibiting the PI3K/AKT/mTOR signaling pathway. Exp Cell Res. 2019;378:198-205.
6. Rota C, Morigi M, Imberti B. Stem cell therapies in kidney diseases: progress and challenges. Int J Mol Sci. 2019;20:2790.

7. Quijada P, Salunga HT, Hariharan N, Cubillo JD, El-Sayed FG, Moshref M, Bala KM, et al. Cardiac stem cell hybrids enhance myocardial repair. Circ Res. 2015:117:695-706.

8. Sun Q, Huang Z, Han F, Zhao M, Cao R, Zhao D, Hong L, et al. Allogeneic mesenchymal stem cells as induction therapy are safe and feasible in renal allografts: pilot results of a multicenter randomized controlled trial. J Transl Med. 2018:16:52

9. Savukinas UB, Enes SR, Sjöland AA, Westergren-Thorsson G. Concise review: the bystander effect: mesenchymal stem cell-mediated lung repair. Stem cells (Dayton, Ohio). 2016;34:1437-44.

10. Aghajani Nargesi A, Lerman LO, Eirin A. Mesenchymal stem cell-derived extracellular vesicles for kidney repair: current status and looming challenges. Stem Cell Res Ther. 2017;8:273.

11. Roushandeh AM, Bahadori M, Roudkenar MH. Mesenchymal stem cell-based therapy as a new horizon for kidney injuries. Arch Med Res. 2017:48:133-46.

12. Mäkelä T, Takalo R, Arvola O, Haapanen H, Yannopoulos F, Blanco R, Ahvenjärvi L, et al. Safety and biodistribution study of bone marrowderived mesenchymal stromal cells and mononuclear cells and the impact of the administration route in an intact porcine model. Cytotherapy. 2015;17:392-402.

13. Wang S, Guo L, Ge J, Yu L, Cai T, Tian R, Jiang Y, et al. Excess integrins cause lung entrapment of mesenchymal stem cells. Stem cells (Dayton, Ohio). 2015;33:3315-26.

14. El-Magd MA, Mohamed Y, El-Shetry ES, Elsayed SA, Abo Gazia M, AbdelAleem GA, Shafik NM, et al. Melatonin maximizes the therapeutic potential of non-preconditioned MSCs in a DEN-induced rat model of HCC. Biomed Pharmacother. 2019;114:108732.

15. Li X, Shang B, Li YN, Shi Y, Shao C. IFNy and TNFa synergistically induce apoptosis of mesenchymal stem/stromal cells via the induction of nitric oxide. Stem Cell Res Ther. 2019:10:18

16. Zhao L, Hu C, Zhang P, Jiang H, Chen J. Novel preconditioning strategies for enhancing the migratory ability of mesenchymal stem cells in acute kidney injury. Stem Cell Res Ther. 2018;9:225.

17. Zhao L, Hu C, Zhang $\mathrm{P}$, Jiang H, Chen J. Melatonin preconditioning is an effective strategy for mesenchymal stem cell-based therapy for kidney disease. J Cell Mol Med. 2020;24:25-33.

18. Cui L, Guo J, Zhang Q, Yin J, Li J, Zhou W, Zhang T, et al. Erythropoietin activates SIRT1 to protect human cardiomyocytes against doxorubicin-induced mitochondrial dysfunction and toxicity. Toxicol Lett. 2017;275:28-38.

19. Jun JH, Jun NH, Shim JK, Shin EJ, Kwak YL. Erythropoietin protects myocardium against ischemia-reperfusion injury under moderate hyperglycemia. Eur J Pharmacol. 2014;745:1-9.

20. Zhou S, Liu YG, Zhang Y, Hu JM, Liu D, Chen H, Li M, et al. Bone mesenchymal stem cells pretreated with erythropoietin enhance the effect to ameliorate cyclosporine A-induced nephrotoxicity in rats. J Cell Biochem. 2018;119:8220-32.

21. Sui BD, Hu CH, Liu AQ, Zheng CX, Xuan K, Jin Y. Stem cell-based bone regeneration in diseased microenvironments: challenges and solutions. Biomaterials. 2019:196:18-30.

22. Grange C, Skovronova R, Marabese F, Bussolati B. Stem Cell-derived extracellular vesicles and kidney regeneration. Cells. 2019;8:1240.

23. Witwer KW, Van Balkom BWM, Bruno S, Choo A, Dominici M, Gimona $M$, Hill AF, et al. Defining mesenchymal stromal cell (MSC)-derived small extracellular vesicles for therapeutic applications. J Extracellular Vesicles. 2019;8:1609206

24. Zhao L, Hu C, Zhang $\mathrm{P}$, Jiang H, Chen J. Preconditioning strategies for improving the survival rate and paracrine ability of mesenchymal stem cells in acute kidney injury. J Cell Mol Med. 2019:23:720-30.

25. Cai J, Jiao $X$, Zhao S, Liang $Y$, Ning $Y$, Shi $Y$, Fang $Y$, et al. Transforming growth factor- $\beta 1$-overexpressing mesenchymal stromal cells induced local tolerance in rat renal ischemia/reperfusion injury. Cytotherapy. 2019:21:535-45.

26. Bi B, Guo J, Marlier A, Lin SR, Cantley LG. Erythropoietin expands a stromal cell population that can mediate renoprotection. Am J Physiol Renal Physiol. 2008;295:F1017-22. 
27. Feng J, Wang W. Hypoxia pretreatment and EPO-modification enhance the protective effects of MSC on neuron-like PC12 cells in a similar way. Biochem Biophys Res Commun. 2017:482:232-8.

28. Lin $H$, Luo $X$, Jin B, Shi H, Gong $H$. The effect of EPO gene overexpression on proliferation and migration of mouse bone marrow-derived mesenchymal stem cells. Cell Biochem Biophys. 2015;71:1365-72.

29. Harting MT, Jimenez F, Xue H, Fischer UM, Baumgartner J, Dash PK, Cox CS. Intravenous mesenchymal stem cell therapy for traumatic brain injury. J Neurosurg. 2009:110:1189-97.

30. Fischer UM, Harting MT, Jimenez F, Monzon-Posadas WO, Xue H, Savitz $\mathrm{SI}$, Laine GA, et al. Pulmonary passage is a major obstacle for intravenous stem cell delivery: the pulmonary first-pass effect. Stem Cells Dev. 2009;18:683-92.

31. Lipowsky HH, Bowers DT, Banik BL, Brown JL. Mesenchymal stem cell deformability and implications for microvascular sequestration. Ann Biomed Eng. 2018;46:640-54.

32. Nystedt J, Anderson H, Tikkanen J, Pietilä M, Hirvonen T, Takalo R, Heiskanen $A$, et al. Cell surface structures influence lung clearance rate of systemically infused mesenchymal stromal cells. Stem Cells (Dayton, Ohio). 2013;31:317-26.

33. Hong T, Ge Z, Meng R, Wang H, Zhang P, Tang S, Lu J, et al. Erythropoietin alleviates hepatic steatosis by activating SIRT1-mediated autophagy. Biochim Biophys Acta. 2018;1863:595-603.

34. Sun W, Qiao W, Zhou B, Hu Z, Yan Q, Wu J, Wang R, et al. Overexpression of Sirt1 in mesenchymal stem cells protects against bone loss in mice by FOXO3a deacetylation and oxidative stress inhibition. Metabolism. 2018;88:61-71.

35. Yoon DS, Choi Y, Lee JW. Cellular localization of NRF2 determines the selfrenewal and osteogenic differentiation potential of human MSCs via the P53-SIRT1 axis. Cell Death Dis. 2016;7:e2093.
36. Mai WX, Gosa L, Daniels WW, Ta L, Tsang JE, Higgins B, Gilmore WB, et al. Cytoplasmic p53 couples oncogene-driven glucose metabolism to apoptosis and is a therapeutic target in glioblastoma. Nat Med. 2017;23:1342-51.

37. Conrad E, Polonio-Vallon T, Meister M, Matt S, Bitomsky N, Herbel C, Liebl M, et al. HIPK2 restricts SIRT1 activity upon severe DNA damage by a phosphorylation-controlled mechanism. Cell Death Differ. 2016;23:110-22.

38. Lin XL, Li K, Yang Z, Chen B, Zhang T. Dulcitol suppresses proliferation and migration of hepatocellular carcinoma via regulating SIRT1/p53 pathway. Phytomedicine. 2020;66:153112.

39. Wang H, Lv C, Gu Y, Li Q, Xie L, Zhang H, Miao D, et al. Overexpressed Sirt1 in MSCs promotes dentin formation in Bmi1-deficient mice. J Dent Res. 2018;97:1365-73.

40. Wang Y, Chen G, Yan J, Chen X, He F, Zhu C, Zhang J, et al. Upregulation of SIRT1 by kartogenin enhances antioxidant functions and promotes osteogenesis in human mesenchymal stem cells. Oxid Med Cell Longev. 2018;2018:1368142

41. Liu X, Chen H, Zhu W, Chen H, Hu X, Jiang Z, Xu Y, et al. Transplantation of SIRT1-engineered aged mesenchymal stem cells improves cardiac function in a rat myocardial infarction model. J Heart Lung Transplant. 2014;33:1083-92.

42. Zhou L, Chen X, Liu T, Gong Y, Chen S, Pan G, Cui W, et al. Melatonin reverses $\mathrm{H}_{2} \mathrm{O} 2$-induced premature senescence in mesenchymal stem cells via the SIRT1-dependent pathway. J Pineal Res. 2015;59:190-205.

\section{Publisher's Note}

Springer Nature remains neutral with regard to jurisdictional claims in published maps and institutional affiliations.
Ready to submit your research? Choose BMC and benefit from:

- fast, convenient online submission

- thorough peer review by experienced researchers in your field

- rapid publication on acceptance

- support for research data, including large and complex data types

- gold Open Access which fosters wider collaboration and increased citations

- maximum visibility for your research: over $100 \mathrm{M}$ website views per year

At BMC, research is always in progress.

Learn more biomedcentral.com/submissions 